\title{
Die Definition von Tracern im QSR-Verfahren
}

\section{Günster}

Qualitätsmanagement

Schlüsselwörter

Ergebnisqualität

Dracer

Routinedaten

Medizinische Versorgung

Key words

outcomes of care

Otracer

administrative data

medical care
Institut

Wissenschaftliches Institut der AOK (WIdO)

\section{Bibliografie}

DOI 10.1055/s-0028-1085594 Dtsch Med Wochenschr 2008; 133: S150 - (c) Georg Thieme Verlag KG Stuttgart · New York . ISSN 0012-0472

\section{Korrespondenz} Christian Günster Forschungsbereichsleiter Integrierte Analysen Wissenschaftliches Institut der AOK (WIdO) Kortrijker Straße 1 D-53177 Bonn

Tel. +49 (228) 843-128 eMail christian.guenster@ wido.bv.aok.de
In dem Projekt Qualitätssicherung der stationären Versorgung mit Routinedaten (QSR) wurde ein Verfahren zur Messung von Ergebnisqualität in Krankenhäusern auf der Basis von administrativen Routinedaten entwickelt. Gemeinsam mit den Projektpartnern [AOK-Bundesverband, HELIOS Kliniken, Forschungs- und Entwicklungsinstitut für das Gesundheitswesen in Sachsen Anhalt (FEISA) und Wissenschaftliches Institut der AOK (WIdO)] wurden 8 Tracer, zugehörige Qualitätsindikatoren und Risikoadjustierungsverfahren definiert und im März 2007 veröffentlicht [1]. Im vorliegenden Beitrag wird die Auswahl und Definition von Tracern und zugehörigen Qualitätsindikatoren im QSR-Verfahren dargestellt und mit Blick auf die internationale Literatur zum Thema begründet.

Für eine sinnvolle Auswahl von Tracern werden zumeist folgende Kriterien genannt:

- High volume: Besonders häufige, aber auch besonders typische Erkrankungsbilder in einem interessierenden Fachgebiet. Ähnlich lautende Kriterien sind epidemiologische Relevanz

- Ökonomische Relevanz

- High risk: Besonders risikoreiche Krankheitsbilder oder Prozeduren, bei denen eine hohe, durch medizinische Maßnahmen beeinflussbare Sterblichkeit oder Komplikationsrate bekannt ist.

- Problem prone: Krankheitsbilder oder Prozeduren, bei denen Probleme in der Gesamtversorgung bereits bekannt sind

- Berücksichtigung verschiedener Fachdisziplinen

- Qualität durch geeignete Indikatoren abbildbar

- Adäquate Möglichkeit zur Risikoadjustierung

- Unabhängigkeit vom Vergütungssystem

Ausgehend von diesen Kriterien wurden zunächst 8 Tracer definiert:

Herzinfarkt, Herzinsuffizienz, Schlaganfall / Intrazerebrale Blutung, Kolon- bzw. Rektumoperation bei kolorektalem Karzinom, laparoskopische und offene Appendektomie, Hüftgelenks-Endoprothese bei Hüftfraktur, Hüftgelenks-Totalendoprothese bei Coxarthrose und Kniegelenks-Totalendoprothese.

Zur Definition wurde gemäß den genannten Kriterien von ICD und OPS-Definitionen ausgegangen, z.T. untypische Altersgruppen ausgeschlossen und auf eine DRG-basierte Definition verzichtet.
Grundlage der Tracerauswertung sind die elektronisch von den Krankenhäusern übermittelten Abrechnungsdaten. Sie enthalten Angaben über Erkrankungen und Eingriffe, Liegezeiten und Verlegungen. Der besondere Charme des QSRVerfahrens besteht in der konsequenten fallübergreifenden Analyse dieser Daten in Verbindung mit weiteren administrativen Versichertendaten der Krankenkasse. So werden neben der Krankenhaussterblichkeit auch Sterblichkeiten nach 30 Tagen, 90 Tagen und einem Jahr nach der Krankenhausbehandlung ausgewiesen. Neben Komplikationen im Erstaufenthalt werden komplikationsbedingte Wiederaufnahmen - im gleichen aber auch einem beliebigen anderen Krankenhaus - in den Blick genommen. Beispielsweise liegen für endoprothetische Implantationseingriffe Revisionsraten im Ein-Jahres-Follow Up und im Prinzip auch für mehrjährige Zeiträume vor.

Kernbestandteil des QSR-Projekts ist der QSRKlinikbericht. Der Bericht enthält risikoadjustierte Qualitätsmessungen für mehrere konservativ und chirurgisch-interventionell behandelte Indikationen. Er kann für jedes deutsche Krankenhaus - eine statistische Mindestanzahl an AOKPatienten vorausgesetzt - erstellt werden. Die Ergebnisse der einzelnen Klinik werden den Erwartungswerten gegenübergestellt, die anhand aller bundesweit durchgeführten Behandlungen berechnet werden. Die Klinikwerte werden mit den Referenzwerten auf Bundesebene und in einem Ranking mit den Ergebnissen anderer Kliniken verglichen. Trendanalysen geben der Klinik Auskunft über die zeitliche Entwicklung der Klinikperformance.

Weitere Tracer, wie Geburtshilfe oder Linksherzkatheter bzw. perkutane koronare Intervention sind in Vorbereitung.

Autorenerklärung: Der Autor arbeitet für das WIdO und den AOK-Bundesverband am Projekt „Qualitätssicherung der stationären Versorgung mit Routinedaten“.

Literatur

1 AOK-Bundesverband, Forschungs- und Entwicklungsinstitut für das Sozial- und Gesundheitswesen Sachsen-Anhalt (FEISA), HELIOS Kliniken, Wissenschaftliches Institut der AOK (WIdO) (Hrsg.). Qualitätssicherung der stationären Versorgung mit Routinedaten (QSR). Abschlussbericht. Bonn: 2007http://wido.de/fileadmin/wido/downloads/ pdf_krankenhaus/wido_kra_qsrabschlussbericht_0407.pdf 